\title{
Structural, magnetic and dielectric properties of ultrafine nickel substituted cobalt ferrite- reduced graphene oxide nanocomposites
}

\section{N. Ambikeswari}

Carbon Nanomaterials Laboratory, Department of Physics, National Institute of Technology, Tiruchirappalli

\section{S. Manivannan ( $\nabla$ ksmaniphysics@yahoo.com )}

Carbon Nanomaterials Laboratory, Department of Physics, National Institute of Technology, Tiruchirappalli

\section{Research Article}

Keywords: Graphene oxide, dielectric properties, ferrites, magnetic properties, superparamagnetism.

Posted Date: August 17th, 2020

DOI: https://doi.org/10.21203/rs.3.rs-58780/v1

License: (a) (i) This work is licensed under a Creative Commons Attribution 4.0 International License. Read Full License

Version of Record: A version of this preprint was published at Journal of Electronic Materials on August 8th, 2021. See the published version at https://doi.org/10.1007/s11664-021-09130-0. 


\section{Abstract}

Nickel substituted cobalt ferrite - reduced graphene oxide (NCF-RGO) composite was synthesized using a facile one-pot chemical co-precipitation method. The addition of graphene oxide (GO) leads to the dimensional decrease of NCF nanoparticles $(<10 \mathrm{~nm})$ in the composite, which controls the magnetic and dielectric properties. Nickel with lower ionic radius, compared to cobalt and its substitution in the composite along with the incorporation of $\mathrm{GO}$ results in the transition of ferromagnetic to superparamagnetic ordering. A large magnetisation of $51.63 \mathrm{emu} / \mathrm{g}$ and a high dielectric constant of 3.92 x $106($ at $100 \mathrm{~Hz}$ ) were also developed from the composite. The conjugated mechanism of magnetic combined electrical property, with a high charge-storing capability and tuneable magnetization, enables its potential use in magnetic flipping or switching devices and data storing systems.

\section{Introduction}

Dielectric deals with materials having desirable permittivity; low loss and good processability find their unique applications in high $k$-gate dielectrics and data storage devices. In principle, the substantial lattice vibrations displace the intrinsic dipole moment and thus results in dielectric permittivity. Several factors such as morphology, contribution of grain boundaries, and the internal material layers are said to be the deciding factors responsible for the dielectric properties. In addition to, extrinsic effects such as the interfacial polarization of highly delocalized electrons at the grain boundaries formulates the orientation of electric dipoles [1].

Ferrites, in general exhibit high magnetic property with large magnetisation and likely to get modifed with the grafting of suitable substitutions. Transition metal ferrites, $\mathrm{MFe}_{2} \mathrm{O}_{4}(\mathrm{M}=\mathrm{Co}, \mathrm{Ni}, \mathrm{Mn}, \mathrm{Zn}, \mathrm{Fe}$, etc., $)$ crystallizes in an inverse spinel structure with a general formula for the ion distribution $\mathrm{A}^{3+}\left[\mathrm{B}^{2+} \mathrm{B}^{3+} \mathrm{O}_{4}{ }^{2-}\right.$ where $A$ denotes tetrahedral cation sites and $B$ denotes octahedral cation sites [2]. The tetrahedral A-sites are occupied by half of the $\mathrm{Fe}^{3+}$ cations, while the octahedral $\mathrm{B}$-sites are occupied by the rest of the $\mathrm{Fe}^{3+}$ cations and the divalent $\mathrm{M}^{2+}$ cations. The interaction between $\mathrm{Fe}^{3+}$ and $\mathrm{M}^{2+}$ ions or a combination of both has a huge impact on their electric and magnetic properties. The dielectric properties of ferrites strongly dependent on the distribution of cations, the required conditions for its preparations and the substitution of various transition metals [2].

Substitutional or doped ferrites, such as $\mathrm{CoFe}_{2-x} \mathrm{MnO}_{4}$ and $\mathrm{Ni}_{0.95-x} \mathrm{Co}_{x} \mathrm{Cu}_{0.05} \mathrm{Fe}_{2} \mathrm{O}_{4}$ produced a dielectric constant of $10^{3}$ and $10^{2}$, respectively at $27^{\circ} \mathrm{C}$ with the contribution of different transitional metals together in the compound $[3,4]$. In a similar manner, $\mathrm{Co}_{1-x} \mathrm{Ni}_{x} \mathrm{Fe}_{2} \mathrm{O}_{4}$ came into action with varying concentrations of $\mathrm{Ni}$ and $\mathrm{Co}$ and displayed a dielectric constant of $10^{3}$ [5]. However, the aforementioned ferrites are limited in data storing applications due to their comparatively low-permittive nature. On the other hand, certain other ferrites such as $\mathrm{BiFeO}_{3}$ and $\mathrm{NiCrFeO}_{4}$ exhibit a high temperature dielectric constant in the order of $10^{4}$ and $10^{6}$, respectively, at $100 \mathrm{~Hz}$, based on polaron hopping mechanism $[6,7]$. These ferrites are hence nrohihited due to its high-temperature dependency, as room temperature Loading [MathJax]/jax/output/CommonHTML/jax.js 
properties are a prime requisite in many storage applications. Therefore, our aim is to produce a hybrid composite material that involves: (i) a matrix with a large surface area that anchors any functional moieties within it and with high conductivity capable of transferring better charge mobility, in turn renders high permittivity and, (ii) a magnetic filler with a large magnetisation, highly reactive and are able to change itself physically and chemically, promoting high permeability. The combination of a highly dielectric and magnetic properties in a single entity or composite could favour its entry in data storage applications.

In the event of preparing hybrid composites, we opted graphene oxide (GO) as a matrix, to arrest the nanofillers, due to its extraordinary and demanding properties. GO with its ample number of oxygenated functional groups, regarded as a semimetal, has the ability to orient the dipoles, along a particular direction, which accounts for the polarization to take place. On the other hand, we selected ferrites as fillers and among them cobalt ferrite stands dominant and remains to be a robust ferromagnetic material that performs its best role in the flourishing fields of data storage systems $[8,9]$. Incorporation of a similar dopant like nickel in it, finds a better pathway to elucidate the storage capability, because a number of active grains from cobalt and nickel along with Fe ions, contribute to the overall dielectric properties $[5,10]$.

The addition of GO to the ferrites adds insight and usability in the vast areas of temporary memory devices. Similar GO/RGO-ferrite composites have been already reported in which the dielectric and magnetic properties were analysed. Among them the composites made from $\mathrm{GO}$ and $\mathrm{Zn}_{\mathrm{x}} \mathrm{Fe}_{1-\mathrm{x}} \mathrm{Fe}_{2} \mathrm{O}_{4}$ produces ultrafine ferrite nanoparticles that enhanced the dielectric constant upto $10^{2}$ and exhibited a ferromagnetic property in it [11]. Likewise, $\mathrm{RGO}-\mathrm{La}_{0.9} \mathrm{Bi}_{0.1} \mathrm{FeO}_{3}$ composites with ferromagnetic ordering, synthesized via sol-gel method, also delivered a dielectric constant of $10^{5}$ [12]. Yet another material, Co-Ni doped $\mathrm{Ca}$ ferrite $\left(\mathrm{Ca}_{2} \mathrm{Co}_{1.5} \mathrm{Ni}_{0.5} \mathrm{Fe}_{12} \mathrm{O}_{22}\right)$ prepared via sol-gel technique, when composited with $\mathrm{GO}$, increased its dielectric permittivity from $10^{1}$ to $10^{3}$. Moreover it was reported that the dielectric constant increases with the increase in GO loading [13].

Meanwhile, superparamagnetism is an inherent and unique characteristic behaviour of the ferrites. Compared to bulk form, smaller sized particles in the magnetic single domain state have tremendous variations in the properties of the ferrites. Moreover, the magneto-crystalline anisotropy plays a key role in the contribution of magnetic moments and also controls the formation of nano domain states [14]. The incorporation of a non-magnetic substance to the ferrite community, in controlled concentration, also find chances to form single domains, rather multi-domains states promoting superparamagnetic property of the mixture. Considering the aforesaid fact, the present composite, nickel substituted cobalt ferrite (NCF) - reduced graphene oxide (RGO), contains substituted ferrites capable of readily creating nanodomains with the inclusion of a non-magnetic entity such as RGO that provides space for the existence of superparamagnetism in the material.

In the previous work, we discussed the magnetodielectric properties of cobalt ferrite - graphene oxide (CFLoading [MathJax]/jax/output/CommonHTML/jax.js the applied magnetic field and dielectric properties were 
studied. Here we aimed to study the dielectric mechanism, with the application of an A.C. electric field. Moreover, the inclusion of $\mathrm{Ni}$ along with the grafting of RGO, reduces the particle size of NCF nanoparticles, allowing more surface charge carriers, thus favours high dielectricity in the composite. Therefore, the as-synthesized, NCF - RGO composite has a magnetic flexibility in its property, allowing ease in magnetisation and demagnetisation. This enables its potential usage in magnetic switching systems and portable memory storage applications.

\section{Experimental Details}

GO was synthesized via modified Hummers' method from graphite powder [16]. CF was synthesized by using the procedure already reported by us [15]. A similar procedure was adopted for NCF, except for, half the amount of $0.4 \mathrm{~g}$ of cobalt chloride is substituted with nickel chloride. Thus, NCF mixture was synthesized from $0.2 \mathrm{~g}$ of nickel chloride and $0.2 \mathrm{~g}$ of cobalt chloride, respectively, with $0.92 \mathrm{~g}$ of ferric chloride as precursors. The corresponding composites such as CF-RGO and NCF-RGO were also synthesized, with the addition of $100 \mathrm{mg}$ of GO, to the aforementioned reactants. Here, a one-pot chemical co-precipitation method was adopted, to fetch out the said composites, where GO, eventually gets reduced into RGO.

All the synthesized samples including ferrites and their composites were washed with copious amount of double distilled water and ethanol, using vacuum filtration technique with the aid of isopore polycarbonate filter membranes, having a pore size of $3 \mu \mathrm{m}$. The filtered content was then dried at $60^{\circ} \mathrm{C}$ and ground into fine powders. These powdered samples were then made into pellets with the aid of pelletizer, having a dimension of $1 \mathrm{~mm}$ thick and $8 \mathrm{~mm}$ diameter. The ohmic contacts for the as-prepared pellets were made using silver paste on both the sides. These pellets were dried for sufficient time in order to prevent the diffusion of silver ions into them.

A schematic representation of the formation of NCF nanoparticles, anchored onto the RGO sheets, is shown in Scheme 1.

\section{Characterization}

The X-ray diffraction (XRD) pattern was recorded using Rigaku Ultima III X-ray diffractometer, with $\mathrm{CuK}_{\mathrm{a} 1}$ radiation $(1.5406 \AA)$ at a scanning rate of $4^{\circ} \mathrm{min}^{-1}$. The Raman scattering was measured with the aid of LabRAM HR Evolution Raman spectrometer, having an excitation wavelength of $532 \mathrm{~nm}$. The presence of functional groups and their inter-molecular bonding was analyzed using Perkin-Elmer Fourier Transform infrared spectrometer (FT-IR) operating in the range of $4000-400 \mathrm{~cm}^{-1}$. The morphological and microstructural analyses were done using field emission scanning electron microscope (FE-SEM) (Quanta 250 FEG, FEI) and high - resolution transmission electron microscope (HR-TEM) (JEOL JEM-2010, Japan). The magnetisation properties were studied, using 7404 Lakeshore model vibrational sample magnetometer (VSM) at $27^{\circ} \mathrm{C}$. The dielectric measurements for the as-prepared silver coated pellet 


\section{Results And Discussion}

Figure 1(a), shows the X-ray diffraction pattern for CF and NCF, projecting six major patterns around $18.39^{\circ}, 30.12^{\circ}, 35.64^{\circ}, 43.22^{\circ}, 56.25^{\circ}$ and $62.77^{\circ}$ corresponding to (111), (220), (311), (400), (511) and (440), respectively, and found to be consistent with the JCPDS Card No. 22-1086. NCF shows a shift towards higher angles at $57.03^{\circ}$ and $63.11^{\circ}$ correspond to (511) and (440) planes. The shift is due to the smaller ionic radius $(0.069 \mathrm{~nm})$ of $\mathrm{Ni}^{2+}$, than that of $(0.072 \mathrm{~nm}) \mathrm{Co}^{2+}$, with lower lattice parameter and lattice volume [5].

Figure $1(\mathrm{~b})$, represents the X-ray diffraction pattern for GO, CF-RGO and NCF-RGO. The pattern around $10.39^{\circ}$ with the orientation of (001), confirms the formation of well-exfoliated layers of GO, denotes the fully oxidized graphite. The disappearance of $10.39^{\circ}$ pattern in the composites, shows the eventual reduction of $\mathrm{GO}$ into RGO, with the partial removal of the functional groups from it. The formation of RGO was confirmed from the projection of hump at $24.53^{\circ}$ with the orientation of (002).

Figure 2(a), projects a broad peak around $3288 \mathrm{~cm}^{-1}$ for $\mathrm{CF}$, attributed to $\mathrm{O}-\mathrm{H}$ stretching vibration and centered at $3326 \mathrm{~cm}^{-1}$ for NCF. In ferrites, the presence of $\mathrm{O}-\mathrm{H}$ peak arises due to the absorption of water molecules during the synthesis. A sharp peak originated from the Fe-O stretching around $565 \mathrm{~cm}^{-1}$, and no noticeable peaks were present for $\mathrm{Co}-\mathrm{O}$ and $\mathrm{Ni}-\mathrm{O}$ due to weaker bond strength compared to $\mathrm{Fe}-\mathrm{O}$. This indicates the trivalent nature of $\mathrm{Fe}^{3+}$ ions dominate the divalent $\mathrm{Co}^{2+}$ and $\mathrm{Ni}^{2+}$ ions in the mixture [10]. In other words, the disappearance of $\mathrm{Co} / \mathrm{Ni}$ - $\mathrm{O}$ peaks must be due to the ingestion of strong Fe-O vibration peak.

Figure 2(b), shows the FT-IR spectrum of GO, that contains the predominant functional groups in its spectrum. Also in composites, some of the oxygenated groups find their presence around 1155 and $1410 \mathrm{~cm}^{-1}$ that correspond to the $\mathrm{C}-\mathrm{O}$ stretching vibration and tertiary $\mathrm{C}-\mathrm{O}-\mathrm{H}$ deformation bands, respectively. Likewise, the peaks around $1585 \mathrm{~cm}^{-1}$ attributes to the unoxidized carbon backbone of $\mathrm{sp}^{2}$ hybridized aromatic $\mathrm{C}=\mathrm{C}$ bonds and $1722 \mathrm{~cm}^{-1}$ belong to the stretching of $\mathrm{COOH}$ vibrations, respectively. The broad peak centered around $3328 \mathrm{~cm}^{-1}$ ascribed to $\mathrm{O}-\mathrm{H}$ stretching vibrations. It was observed that most of the oxygenated peaks in the composites got overlapped with each other, due to strong intermolecular bonding between them. It was also seen, that the intensity of the peaks was decreased, and even some got diminished, due to the partial removal of functional groups from RGO, in the composite.

The spinel ferrite structure can be described by Fd $3 \mathrm{~m}$ centro-symmetric space group and factor group analysis predicts the existence of five Raman active internal modes: $A_{1 g}+E_{g}+3 T_{2 g}$ [17]. Figure 3(a), presents the active modes of ferrites that finds their existence around $213,283,465,595$ and $681 \mathrm{~cm}^{-1}$ corresponding to $T_{2 g}, E_{g}, T_{2 g}, T_{2 g}$ and $A_{1 g}$ vibrations, respectively. The significance of each vibrational mode is well established in the spectrum. Both $E_{g}$ and $T_{2 g}$ modes correspond to the symmetric stretching Loading [MathJax]/jax/output/CommonHTML/jax.js es, present at the octahedral sites and occurs naturally in the 
low frequency regime (below $500 \mathrm{~cm}^{-1}$ ). While, $A_{1 g}$ mode attributes to anti-symmetric bending vibration of $\mathrm{Fe}^{3+}$ ions present at the tetrahedral sites, which tend to occur at high frequency region (above $500 \mathrm{~cm}^{-}$ ${ }^{1}$ ) [14]. Among the three distinguishable $T_{2 g}$ modes, initial one at lower wavenumbers attributes to the presence of impurity phases in the composite, the middle one corresponds to symmetric stretching vibration, and the last one, usually a shoulder peak of $A_{1 g}$ vibration, belongs to the distribution of local cations at either tetrahedral or octahedral positions [14].

The Raman spectrum of GO in Fig. 3(b), shows the D- band at 1335 and G- band at $1594 \mathrm{~cm}^{-1}$, respectively. The composites also showed five Raman active modes around 217, 285, 459, 598 and $682 \mathrm{~cm}^{-1}$ belonging to $T_{2 g}, E_{g}, T_{2 g}, T_{2 g}$ and $A_{1 g}$ vibrations. A comparative shift towards higher wavenumbers in the $D$ and $G$ bands was also noticed in the composites indicating a reduction of $G 0$ into RGO. It is noteworthy that G-band was observed to have a lower intensity, compared to D-band in NCFRGO. The $I_{D} / I_{G}$ ratio for GO, CF-RGO and NCF-RGO were found to be $1.15,1.19$ and 1.67 , respectively, indicates the presence of defects has been increased in the RGO composite..

Figure 4(a), displays the well exfoliated sheets of GO. Figure 4(b) and (c) shows the highly agglomerated magnetic spherical nanoparticles of ferrites, with a dimension of more than $100 \mathrm{~nm}$. Figure 4(d) and (e) show the existence of RGO in the composites, with a much reduced nanosized ferrites decorated over it. However, the FE-SEM images produced only a prediction about the size of the nanoparticles in the composite. Thus, to study the morphological changes and to define the particle size with better resolution, HR-TEM was adopted as an efficient tool. Figure 5(a) and (b), depict images of highly dispersed nanoparticles on RGO composites. Here, the reduction in the particle size was clearly seen. The average particle sizes of $\mathrm{CF}$ and NCF, in the composites were calculated, and found to be around 8-10 and 6-8 nm, respectively. [Fig. 5(c) and (d)].

The existence of RGO in the composites, serve the ferrite nanoparticles as anchoring sites and also, prevents the agglomeration of the magnetic nanoparticles, during the reaction process [19]. The increased defects that arises in the reduction process from GO to RGO, promotes excess of anions and the presence of $\mathrm{Fe}^{2+}$ and $\mathrm{Fe}^{3+}$ in the ferrites act as cations. Thus, RGO pertain itself as a host in arresting the ferrite nanoparticles and consequently limits the growth and hence reduce the diameter drastically of the latter [20].

Figure 6(a), shows the $\mathrm{M}-\mathrm{H}$ curve for the pristine $\mathrm{GO}$ that exhibits a weak ferromagnetic behaviour having a magnetisation (Ms) of $0.42 \mathrm{emu} / \mathrm{g}$ and $\mathrm{Hc}$ of $64 \mathrm{Oe}$. The magnetism in $\mathrm{GO}$ arises, due to the occurrence of $\mathrm{O}$-atoms bounded to the carbon network and the defects created within them, promotes excess free electrons, that induces magnetic moments. Thus the, so-called induced magnetism in a non- magnetic material has been already reported, and it is also been discussed in our previous work [15,20-22]. It is noteworthy to observe an antiferromagnetic ordering to get produced along the edges of the $\mathrm{M}-\mathrm{H}$ curve, in GO. This was on behalf of the spin reorientation that is exhibited, with an increase in the applied maanetic field that is analoaous to the behaviour of Gd doped Zn-S [23]. 
Figure 6(b), displays the respective magnetisation curves for CF and NCF, in which they exhibit ferromagnetic behaviour. CF reveals a high Ms of 62.57emu/g and a very large Hc of 1462.21 Oe as it contains an inherent strong magnetic property. When the nickel is substituted in CF, there exists a decrease in $\mathrm{Ms}$, due to the contribution of magnetic moments from $\mathrm{Ni}^{2+}$ ions to the material [24]. Since $\mathrm{Ni}$, has low magneto-crystalline anisotropy compared to Co, NCF acquires a decrease in Ms of $57.09 \mathrm{emu} / \mathrm{g}$ and a $\mathrm{Hc}$ of 152.03 Oe. Moreover $\mathrm{Ni}^{2+}$ ions prefers to be in the octahedral B-site, that does not get much influenced by the magnetic interactions, rather $\mathrm{Co}^{2+}$ (tetrahedral $\mathrm{A}$-site) and $\mathrm{Fe}^{3+}$ (both $\mathrm{A}$ and $\mathrm{B}$ sites) ions [14]. Hence, weak magnetic interactions, also favours low Ms and Hc, compared to other ferromagnetic materials.

Likewise, CF-RGO and NCF-RGO exhibit ferromagnetic and superparamagnetic properties, respectively, [Fig. 6(c)]. CF is considered to be a strong ferromagnetic material and it has high magneto-crystalline anisotropy. The inclusion of GO, in CF-RGO, results in a quantitative decrease of Ms (53.97 emu/g) and $\mathrm{Hc}(50.19 \mathrm{Oe})$, without altering the ferromagnetic ordering. Regarding NCF-RGO, the addition of Ni, is due responsible for the superparamagnetism in the composite. $\mathrm{Ni}$, despite being a ferromagnetic material, has a low magneto-crystalline anisotropy due to smaller ionic radius $(0.069 \mathrm{~nm})$ compared to cobalt $(0.072 \mathrm{~nm})$ and iron $(0.074 \mathrm{~nm})$ [24]. This is true in all the cases, where Ni finds its presence or in places where it was doped. Therefore, NCF, containing Ni along, when incorporated with a low-magnetic or nonmagnetic matrix such as GO/RGO, results in the dimensional decrease, with the dramatic reduction in the particle size upto 6-8 nm (evidenced from HR-TEM). This allows forming single domains states instead of multi-domain system, leading to the drastic lessening of coercive field (23.67 $0 \mathrm{e})$ and a considerable decrease in Ms (51.63 emu/g) [25,26]. Thus, a lower coercive field induces superparamagnetism and that is occurring in the composite.

Figure 7(a) and (b), represents the frequency-dependent dielectric constant ( $\left.\varepsilon^{\prime}\right)$ for GO, CF and NCF ferrites. The $\varepsilon^{\prime}$ for GO found to be $1.64 \times 10^{3}$ and for CF and NCF it was $2.24 \times 10^{3}$ and $5.51 \times 10^{3}$, respectively, at $100 \mathrm{~Hz}$. Among them, NCF shows a pretty rise in its $\varepsilon^{\prime}$, can be due to the contribution of both $\mathrm{Co}^{2+}$ and $\mathrm{Ni}^{2+}$ ions. The increase in population of charge carriers at the grain boundaries is responsible for the dielectric polarization to take place at low-frequencies [27]. In addition, the polarization in ferrites is attributed to the exchange interaction between $\mathrm{Fe}^{2+}$ and $\mathrm{Fe}^{3+}$ ions, which in turn produces the local displacement of electrons, in the same direction as that of the applied electric field that causes polarization [12].

Figure 7(c) represents the $\varepsilon^{\prime}$ of the prepared composites and it was found to have a two- to three- order rise and calculated to be $2.43 \times 10^{5}$ and $3.92 \times 10^{6}$ at $100 \mathrm{~Hz}$ for CF-RGO and NCF-RGO, respectively. An order increase of $\varepsilon^{\prime}$ in NCF-RGO was due to the influence of Ni to CF, with the non-magnetic substance RGO in the composite. This heterogeneous mixture said to lessen the particle size of NCF in the composite, rendering superparamagnetism in the composite on one hand and on the other hand leads to enhanced dielectric constant. The more defective RGO (evidenced from $\mathrm{I}_{\mathrm{D}} / \mathrm{I}_{\mathrm{G}}$ ratio is RAMAN analysis) in the rnmnncite enahlec mnre free elentrnnc that consequently creates dipoles at the electrode material Loading [MathJax]/jax/output/CommonHTML/jax.js

Page $7 / 20$ 
interface and hence large $\varepsilon^{\prime}$ [12]. The strong cationic effects from the magnetic moieties, interacts well with the anionic effects arising from the electron- rich RGO, enters into a lock-in mechanism that favors enhanced dielectricity in the material.

Figure 8 , represents the frequency-dependent $A C$ conductivity $\left(\sigma_{A C}\right)$ of the ferrites and their respective composites. Figure $8(a)$ and (b) shows a gradual increase in the $\sigma_{A C}$ values from low frequency to high frequency region, for GO, CF and NCF and were calculated to be $4.75 \times 10^{-5}, 8.13 \times 10^{-5}$ and $3.48 \times 10^{-4}$ $\mathrm{S} / \mathrm{m}$, respectively. Ferrites have cubic closely packed structure, with cations both at the octahedral (B) and at the tetrahedral $(A)$ sites, and therefore, the exchange interaction of charge carriers between $A$ and $B$ sites is more pronounced, compared to the exchange interaction of charge carriers within $B$ and $B$ sites. Besides, at low-frequency, the grain boundaries are more active, which reduces the number of hopping ions such as $\mathrm{Fe}^{2+}$ and $\mathrm{Fe}^{3+}$ in the ferrite series. Concomitantly, at high frequencies and with the application of an AC electric field, the conductive grains become more active, which renders hopping between $\mathrm{Fe}^{2+}$ and $\mathrm{Fe}^{3+}$ ions, termed as hopping-conduction mechanism [5]. This hopping mechanism is highly responsible for the $\mathrm{AC}$ conductivity to occur at high frequencies in ferrites.

Figure $8(\mathrm{c})$, shows the dramatic increase in $\sigma_{\mathrm{AC}}$ for the composites of CF-RGO and NCF-RGO compared to their parent materials. $\sigma_{A C}$ for CF-RGO and NCF-RGO, were calculated to be $5.17 \times 10^{-2}$ and $9.74 \times 10^{-1}$ $\mathrm{S} / \mathrm{m}$, respectively. The possible cause for the high increase in $\sigma_{\mathrm{AC}}$, lies in the reduction of $\mathrm{GO}$ in the synthesis process. In the reduction process, the $\mathrm{sp}^{3}$ - hybridized bonded carbon atoms are converted into $\mathrm{sp}^{2}$ - hybridized, and hence, the $\pi$-electron network consequently gets altered [28]. Thus, our composites comprise more number of active grains, which generate the so-called electronic conduction and the hopping conduction. Similar work has been reported by Khawas et al. (2020), in which they reported RGO$\mathrm{La}_{0.9} \mathrm{Bi}_{0.1} \mathrm{FeO}_{3}$ composite with a $\sigma_{\mathrm{AC}}$ in the order of $10^{-6} \mathrm{~S} / \mathrm{m}$. Likewise, Kavinkumar et al. (2016) reported a $\sigma_{A C}$ in the order of $10^{-3} \mathrm{~S} / \mathrm{m}$ for the GO-BT (barium titanate) nanocomposite [12,28].

Figure 9, describes the electric moduli (M") against frequency for GO, CF, NCF and composites, Fig. 9(a), illustrates theM" spectrum of $\mathrm{GO}$, where a minor peak exist corresponding to the presence of active grains and a sudden intense peak relates to the presence of large insulating grain boundaries [29]. Both the peaks prevails in the frequency region $\left(10^{4}\right.$ to $\left.10^{5} \mathrm{~Hz}\right)$ that implies, $\mathrm{GO}$ to be an electrically insulating material, resulting in low charge carrier mobility and hence large relaxation time. While, CF and NCF displays only a single peak at high frequency region $\left(\geq 10^{5} \mathrm{~Hz}\right)$ that attributes to more of grain boundaries, due to conducting nature of the samples, pertaining to low relaxation time. The presence of RGO in the composites enables more charge carriers to move rapidly over the interface and hence decreases the relaxation time, hence transfers the relaxation peaks towards high-frequency region [Fig. 9(c)].

Figure 10, shows a clear picture of the relaxation process in the Cole-Cole plot, as it inhibits both the real and imaginary components of the electric moduli. Figure 10(a), illustrates a similar behaviour for G0 as Loading [MathJax]/jax/output/CommonHTML/jax.js CF displays a non-Debye behaviour with two semi-circles in 
their spectra [30]. As mentioned earlier, the first semicircle indicates the presence of active grains and the second due to presence of grain boundaries that occurs in the low and high frequency areas, respectively. Figure 10(c), the Cole-Cole plot for the composites, projects a shift towards high-frequency region. It clearly suggests that the composites prevail themselves in the conductive regime, inhibiting more accumulation of charges at the grain boundaries or interfaces with a very low relaxation time.

The beneficial effects of the relaxation time, derived from the relaxation mechanism, of any material, could be applied in formulating mathematical dielectric models that involves the concept of time-domain functions. The variations in the relaxation curves, finds its potential applications in relaxation oscillatortype systems. Moreover, it is also used to investigate the temperature and time dependent rheological properties of stress induced supramolecular polymer fluids and lubricating greases.

\section{Conclusion}

The present work illustrates a simple in-situ chemical co-precipitation method for the synthesis of nickel substituted cobalt ferrite - reduced graphene oxide (NCF-RGO) composite. A high $\varepsilon^{\prime}$ of $3.92 \times 10^{6}$ was achieved for NCF-RGO, an order increase compared to CF-RGO $\left(2.43 \times 10^{5}\right)$, considered to be the best among the transitional metal ferrites and their composites. The inclusion of $\mathrm{Ni}$ with $\mathrm{CF}$, on a nonmagnetic entity as RGO, exhibits superparamagnetism as a result of the dimensional decrease $(<10 \mathrm{~nm})$, in the composite. The $\sigma_{A C}$, measured to be lying in the order of $10^{-1} \mathrm{~S} / \mathrm{m}$, ensures high conducting behaviour of NCF-RGO. and Cole-Cole plot for the composites were observed to be prevailing at highfrequency side, with low relaxation time, that could be applied in dielectric models, relaxation oscillator circuits and to study the rheology of fluids. The reduction in the particle size of NCF nanoferrites thus controls the electric and magnetic properties of the composite, delivers a very high dielectric constant along with the superparamagnetic property, and enables its potential use in the emerging field of portable memory storage devices.

\section{Declarations}

\section{Acknowledgement}

Dr. T. Arun, IOP, Bhubaneswar, India, Dr. A. Justin Josephus, Dr. B. Karthikeyan and Dr. A. Sreekanth, National Institute of Technology, Tiruchirappalli, India, are acknowledged for providing with FE-SEM, VSM, micro-Raman and FT-IR facilities, respectively.

\section{References}

[1] K.S. Kumar, S. Pittala, S. Sanyadanam, P. Paik, A new single/ few layered graphene oxide with a high dielectric constant of 106; contribution of defects and functional groups, RSC Adv. 5 (2015) 14768. 
[2] R.K. Kotnala and J. Shah, Handbook of Magnetic Materials, Ferrite Materials: Nano to Spintronics Regime. 23 (2015) 291.

[3] C.V. Ramana, Y.D. Kolekar, K.K. Bharathi, B. Sinha and K. Ghosh, Correlation between structural, magnetic and dielectric properties of manganese substituted cobalt ferrite, J. Appl. Phys. 144 (2013) 183907.

[4]. R.S. Devan, Y.D. Kolekar and B.K. Chougule, Effect of cobalt substitution on the properties of nickelcopper ferrite, J. Phys.: Condens. Matter. 18 (2006) 9809.

[5] N.B. Velhal, N.D. Patil, A.R. Sheike, N.G. Despande and V.R. Puri, Structural, dielectric and magnetic properties of nickel substituted cobalt ferrite nanoparticles: Effect on nickel substitution, AIP ADVANCES. 5 (2015) 097166.

[6] S-Z. Lu and X. Qi, Magnetic and dielectric of nanostructured $\mathrm{BiFeO}_{3}$ prepared by sol-gel method, J. Am. Ceram. Soc. 1(2014) 12960.

[7] A.K. Sijo, D.P. Dutta, M. Roy and V.D. Sudheesh, Magnetic and dielectric properties of $\mathrm{NiCrFeO}_{4}$ prepared by solution self-combustion method, Mater. Res. Bull. 94 (2017) 154.

[8] J. Li, H. Feng, J. Jiang, Y. Feng, Z. Xu and D.Qian, One-pot synthesis of $\mathrm{CoFe}_{2} \mathrm{O}_{4}$ nanoparticlesreduced graphene oxide nanocomposite with high performance for levodopa sensing, RSC Adv. 5 (2015) 99669.

[9] P.R. Kumar, P. Kollu, C. Santhosh, K.E.V. Rao, D.K. Kim and A.N. Grace,Enhanced properties of porous $\mathrm{CoFe}_{2} \mathrm{O}_{4}$-reduced graphene oxide composites with alginate binders for Li-ion battery applications, New. J. Chem. 38 (2014) 3654.

[10] X. Chen, C. Hou, Q. Zhang, Y. Li, H. Wang, One-step synthesis of Co-Ni ferrite/graphene nanocomposites with controllable magnetic and electrical properties, Mater. Sci. Eng., B. 177 (2012) 1067.

[11] G. Khurana, N. Kumar, S.Koorayattil and R.S. Katiyar, Structural, magnetic, and dielectric properties of graphene oxide/ $\mathrm{Zn}_{\mathrm{x}} \mathrm{Fe}_{1-\mathrm{x}} \mathrm{Fe}_{2} \mathrm{O}_{4}$ composites, J. Appl. Phys.117 (2015) $17 \mathrm{E} 106$.

[12] E.H. El-Khawas, A.A. Azab and A.M. Mansour, Structural, magnetic and dielectric properties of reduced graphene oxide/ $\mathrm{La}_{0.9} \mathrm{Bi}_{0.1} \mathrm{FeO}_{3}$ nanocomposites, Mater Chem Phys. 241 (2020) 122335.

[13] M.M. Saeed, M. Ajmal and M.U. Islam, Synthesis and characterization of Y-type ferrite reinforced graphene oxide, J. Supercon Nov Magn (2020). DOI: 10.1007/s10948-02005502-5

[14] S. G. Gawas, S. S. Meena, P.Bhatt and V. M. S. Verenkar, Nanoscale-driven structural changes and associated superparamaqnetism inmaqnetically diluted Ni-Zn ferrites,Mater. Chem. Fron. 2 (2018) 300. Loading [MathJax]/jax/output/CommonHTML/jax.js 
[15] N. Ambikeswari and S. Manivannan, "Superior magnetodielectric properties of room temperature synthesized superparamagnetic cobalt ferrite - graphene oxide composite" J. Alloys Compd. 763 (2018) 711.

[16] W. S. Hummers and R. E. Offeman, Preparation of graphitic oxide, J. Am. Chem. Soc., 80 (1958) 1339.

[17] C. Himcinschi, I. Vrejoiu, G. Salvan, M. Fronk, A. Talkenberger, Optical and magneto- optical study of nickel and cobalt ferrite epitaxial thin films and submicron structures J. Appl. Phys. 113, (2013) 084101.

[18] J. Wang, S.A. Kondrat, Y.Wang, G.L. Brett, C. Giles, J. K. Bartley, L. Lu, Q. Liu, C.J. Kiley, G.J.Hutchings. Au-Pd nanoparticles dispersed on composite titania/graphene oxide-support as a highly active oxidation catalyst. ACS Catal. 5 (2015) 3575.

[19] H. N. Lim, N. M. Huang, C.H. Chia, I. Harrison, Inorganic nanostructures decorated graphene, Intech open, Advance topics in crystal growth, 12 (2013) 377.

[20] S.K. Sarkara, K.K. Raul, S.S. Pradhan, S. Basu, A. Nayak, Magnetic properties of graphite oxide and reduced graphene oxide, Physica E. 64 (2014) 78.

[21] T. Tang, F. Liu, Y. Liu, X. Li, Q. Xu, Q. Feng, N. Tang, Y. Du, Identifying the magnetic properties of graphene oxide, Appl. Phys. Lett. 104 (2014) 123104. DOI:10.1063/1.4869827.

[22] K. Bagani, A. Bhattacharya, J. Kaur, A.R. Chowdhury, B. Ghosh, M. Sardar, S. Banerjee, Anomalous behaviour of magnetic coercivity in graphene oxide and reduced graphene oxide, J. Appl. Phys. 115 (2014) 023902.

[23] P. Kaur, S. Kumar, C-L. Chen, K-S. Yang, D-H. Wei, C-L. Dong, C. Srivastava, S.M. Rao, Gd doping induced weak ferromagnetic ordering in ZnS nanoparticles synthesized by low temperature coprecipitation technique, Mater Chem Phys. 186 (2017) 24.

[24] F.C. Fonseca, G.F. Goya and R. F. Jardim, Superparamagnetism and magnetic properties of Ni nanoparticles embedded on $\mathrm{SiO}_{2}$, Phys. Rev. B 66 (2002) 104406.

[25] N. Kumar, G. Khuranaa, A. Gaurc, R.K. Kotnalaa, Observation of superparamagnetism in ultra-fine $\mathrm{Zn}_{\mathrm{x}} \mathrm{Fe}_{1-\mathrm{X}} \mathrm{Fe}_{2} \mathrm{O}_{4}$ nanocrystals synthesized by co-precipitation method, Mater Chem Phys. (2012) 1-6.

[26] B. Zou, A. Rondinone, J. Zhang, Z.J. Liu, Chemical control of superparamagnetic properties of magnesium and cobalt spinel ferrite nanoparticles through atomic level magnetic coupling, J. Am. Chem. Soc. $122(2000) 6263$.

[27] C.G. Koops, On the dispersion of resistivity and dielectric constant of some semiconductors at audio Loading [MathJax]/jax/output/CommonHTML/jax.js 
[28] T. Kavinkumar, P. Senthilkumar, S. Dhanushkodi and S. Manivannan, Dielectric transition and ferroelectric properties of graphene oxide- barium titanante composites, J Eur Ceram Soc. 37 (2017) 1401.

[29] T. Kavinkumar, D. Sastikumar and S. Manivannan, Effect of functional groups on dielectric, optical, gas sensing properties of graphene oxide and reduced graphene oxide at room temperature, RSC Adv., 5 (2015), 10816.

[30] W. Wang, B. Xu, P. Gao, W. Zhang, Y. Sun, Electrical and dielectric properties of $\mathrm{HoMnO}_{3}$ ceramics", Solid State Commun.177 (2014).

\section{Figures}
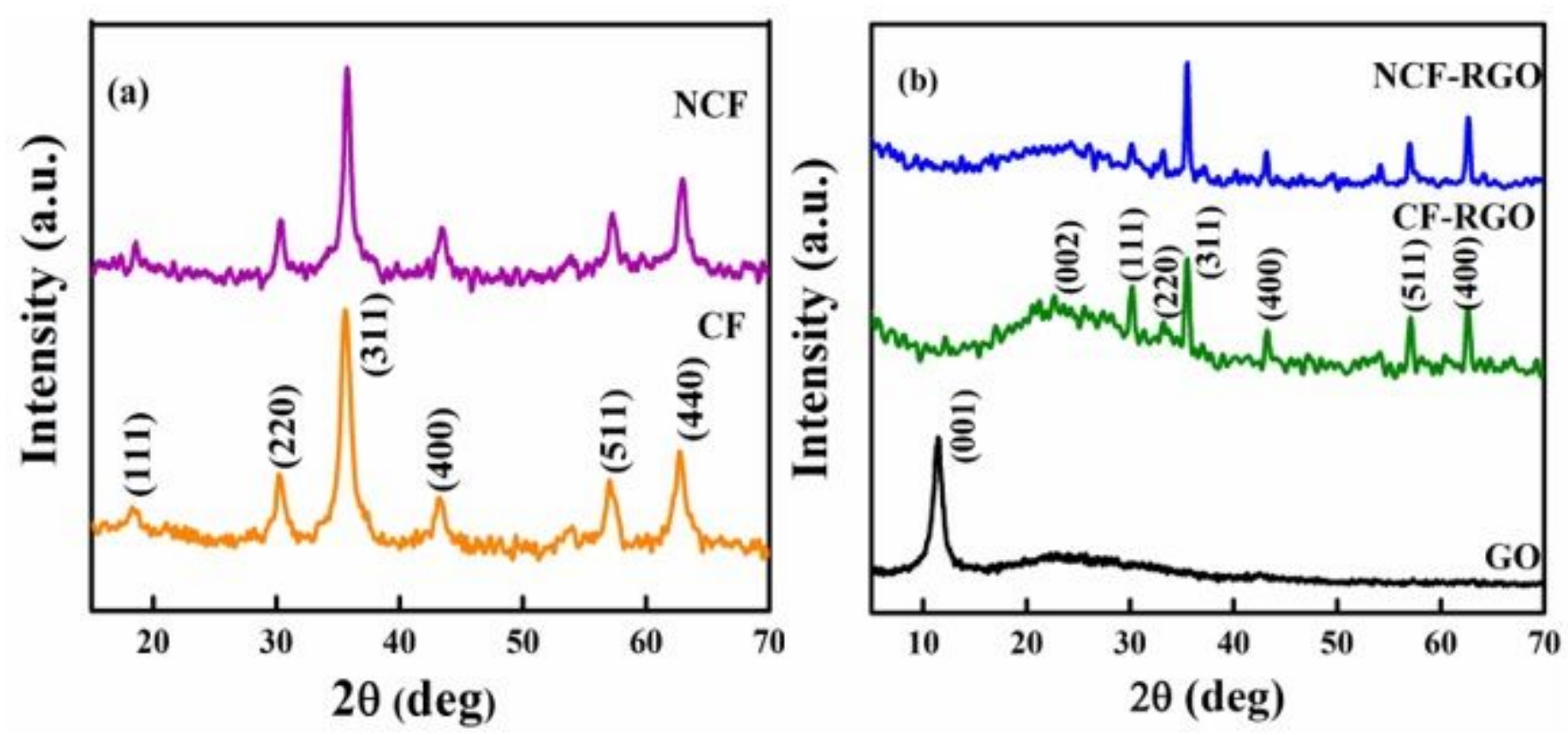

Figure 1

Powder XRD pattern for (a) CF and NCF, (b) GO, CF-RGO and NCF-RGO composites. 

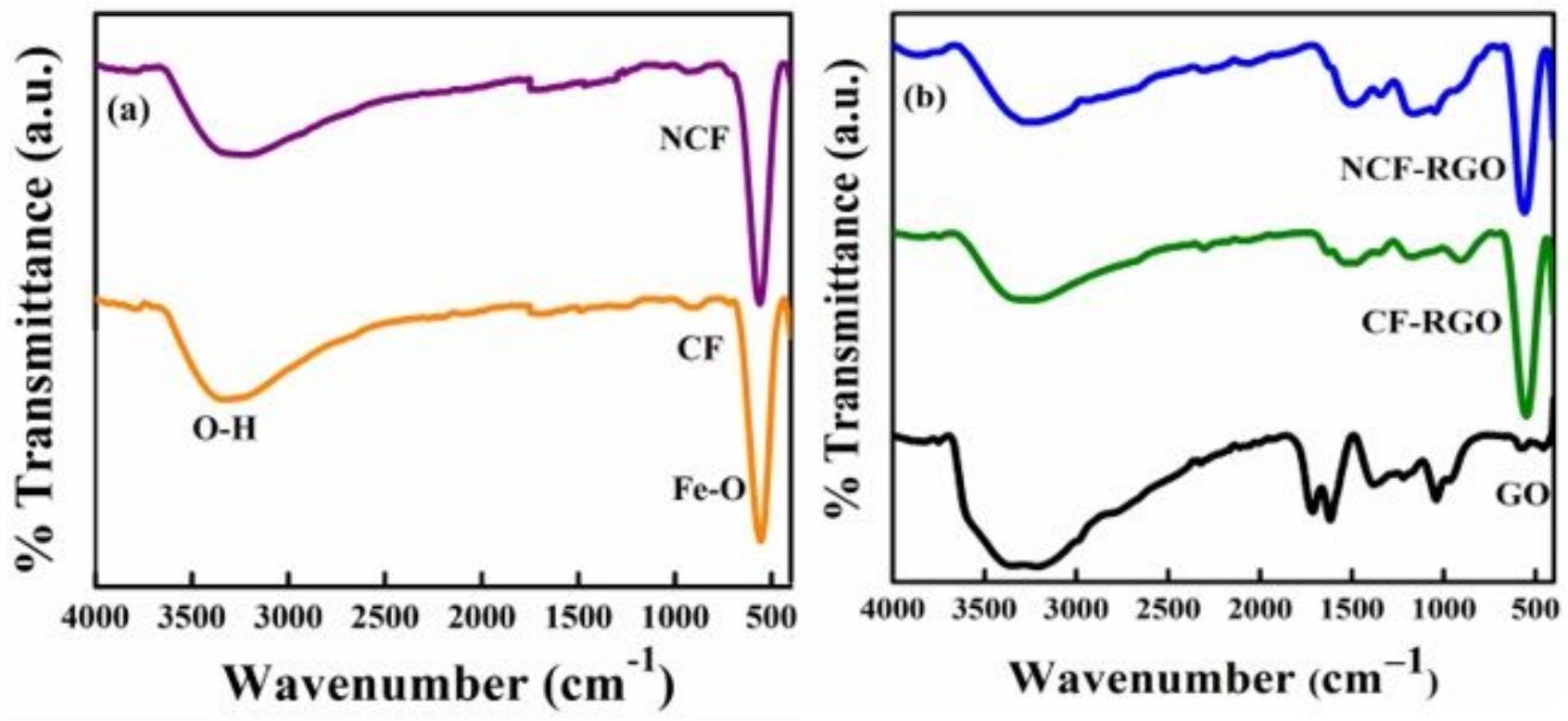

Figure 2

FT-IR spectra for (a) CF and NCF, (b) GO, CF-RGO and NCF-RGO composites.
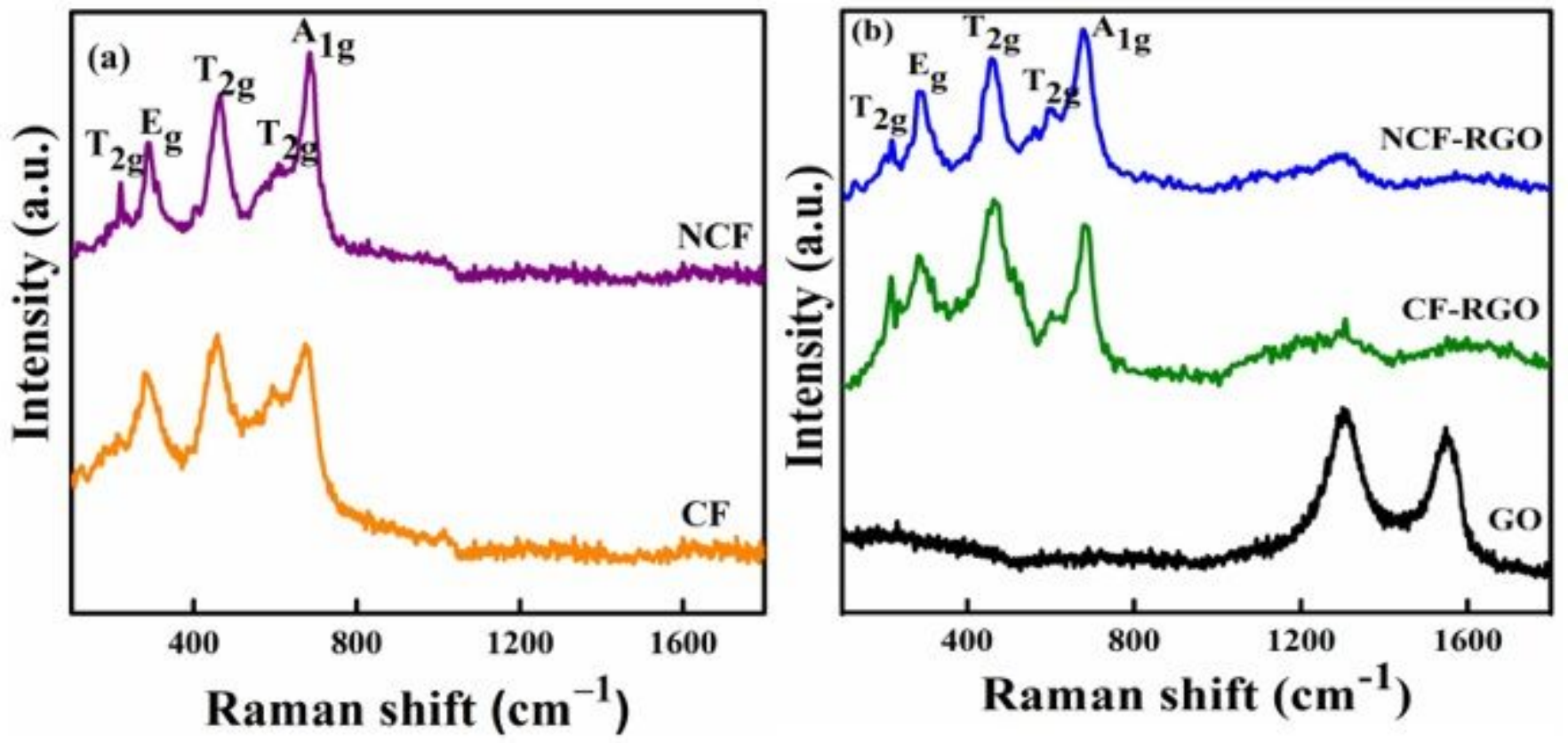

Figure 3

Micro-Raman spectra for (a) CF and NCF, (b) GO, CF-RGO and NCF-RGO composites. 

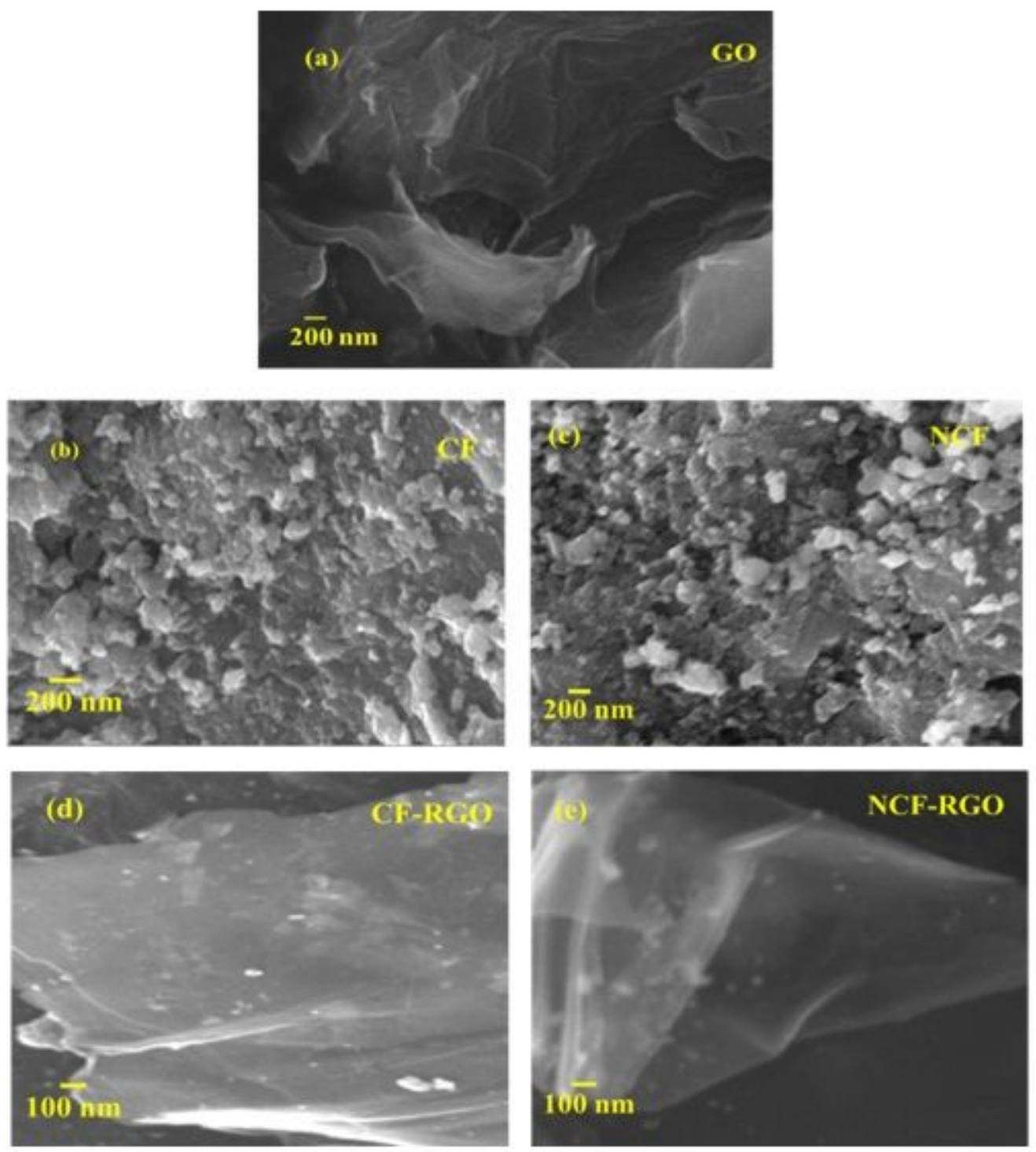

\section{Figure 4}

FE-SEM micrographs for (a) GO, (b) CF, (c) NCF, (d) CF-RGO and (e) NCF-RGO. 

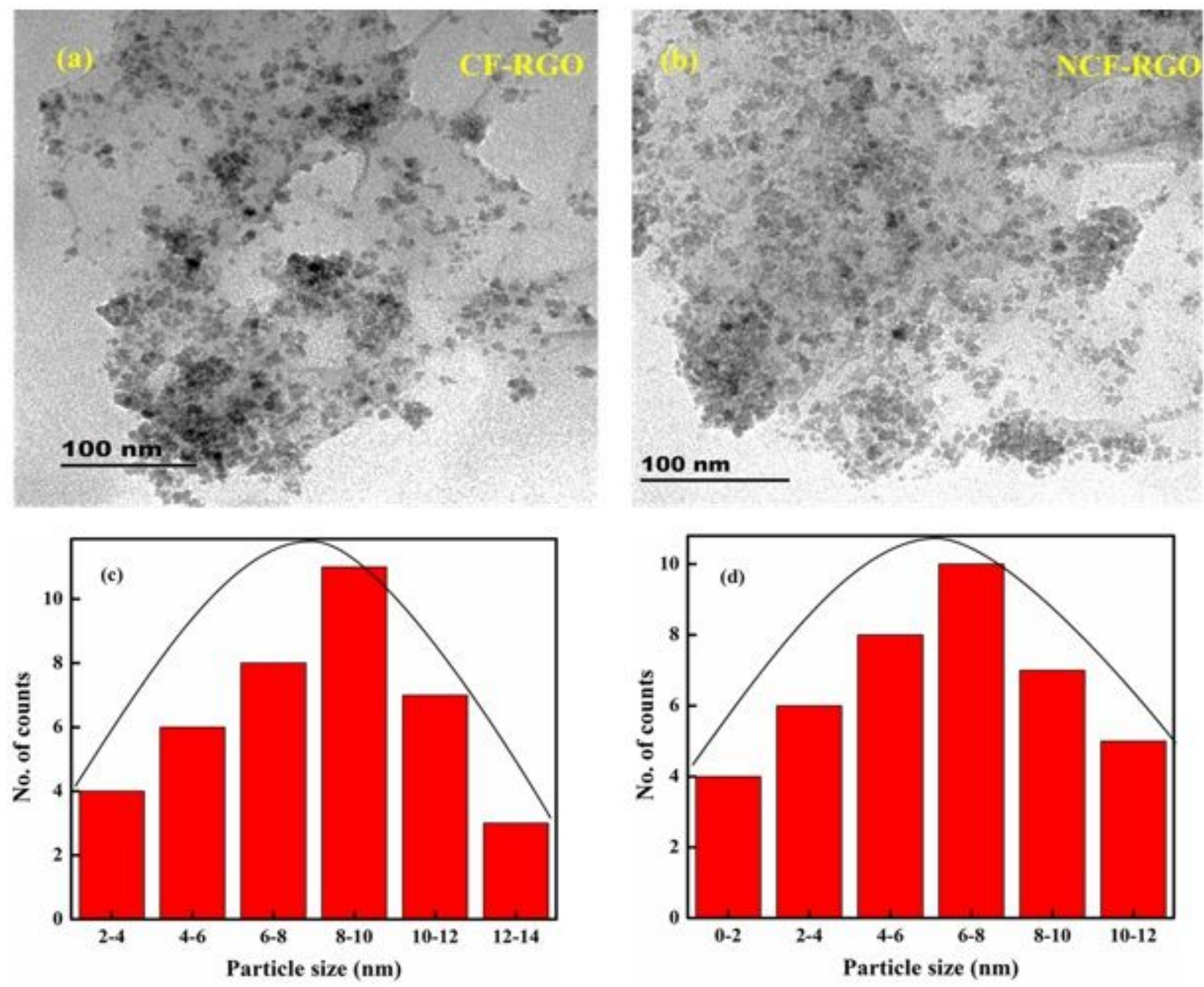

Figure 5

HR-TEM images of (a) CF-RGO and (b) NCF-RGO, histogram for the distribution of particle size in (c) CFRGO and (d) NCF-RGO composites. 

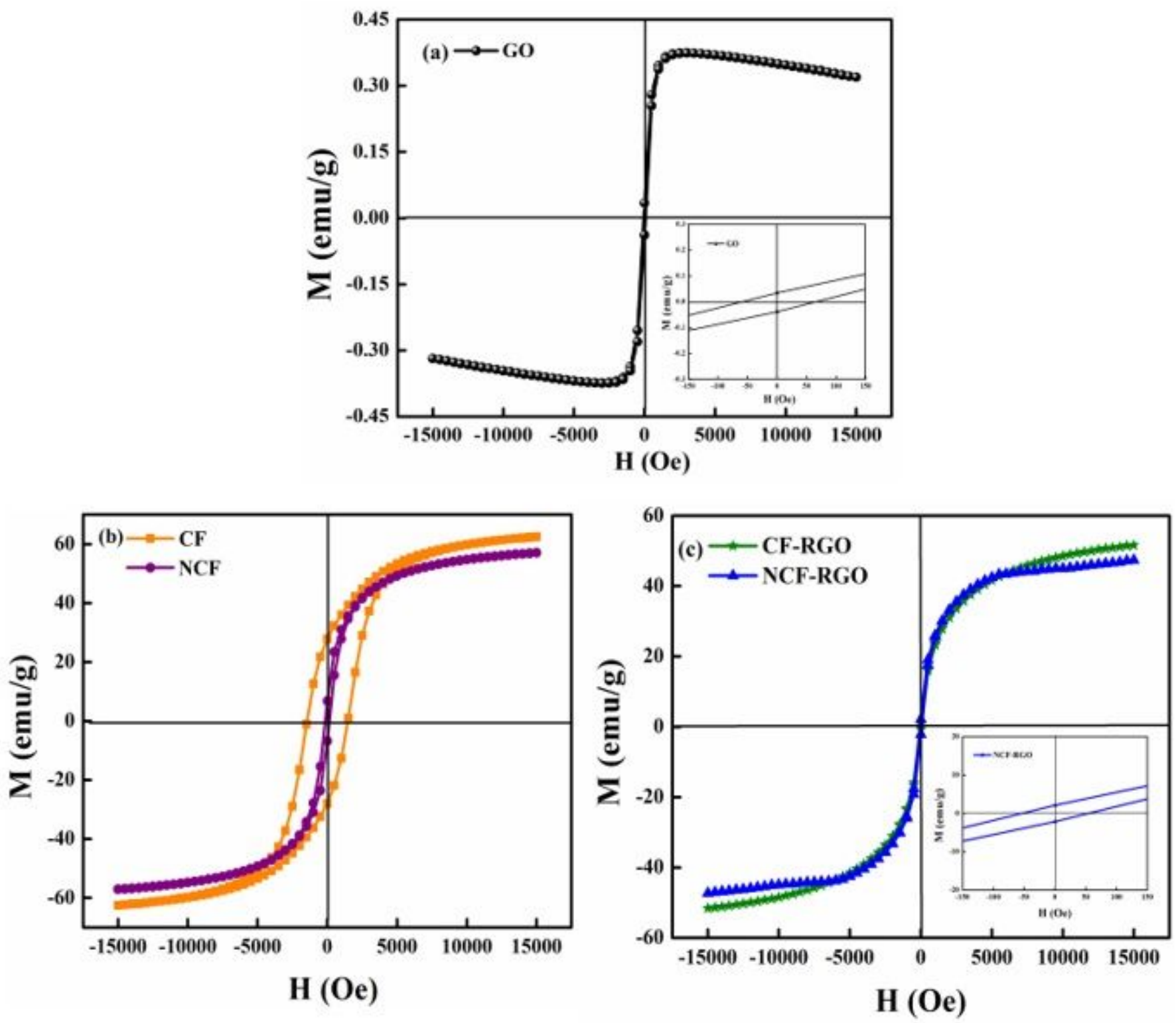

Figure 6

Room temperature M-H curves (a) GO (inset shows the M-H curves at low fields), (b) CF and NCF, (c) CFRGO and NCF-RGO composites (inset shows the M-H curves at low fields). 

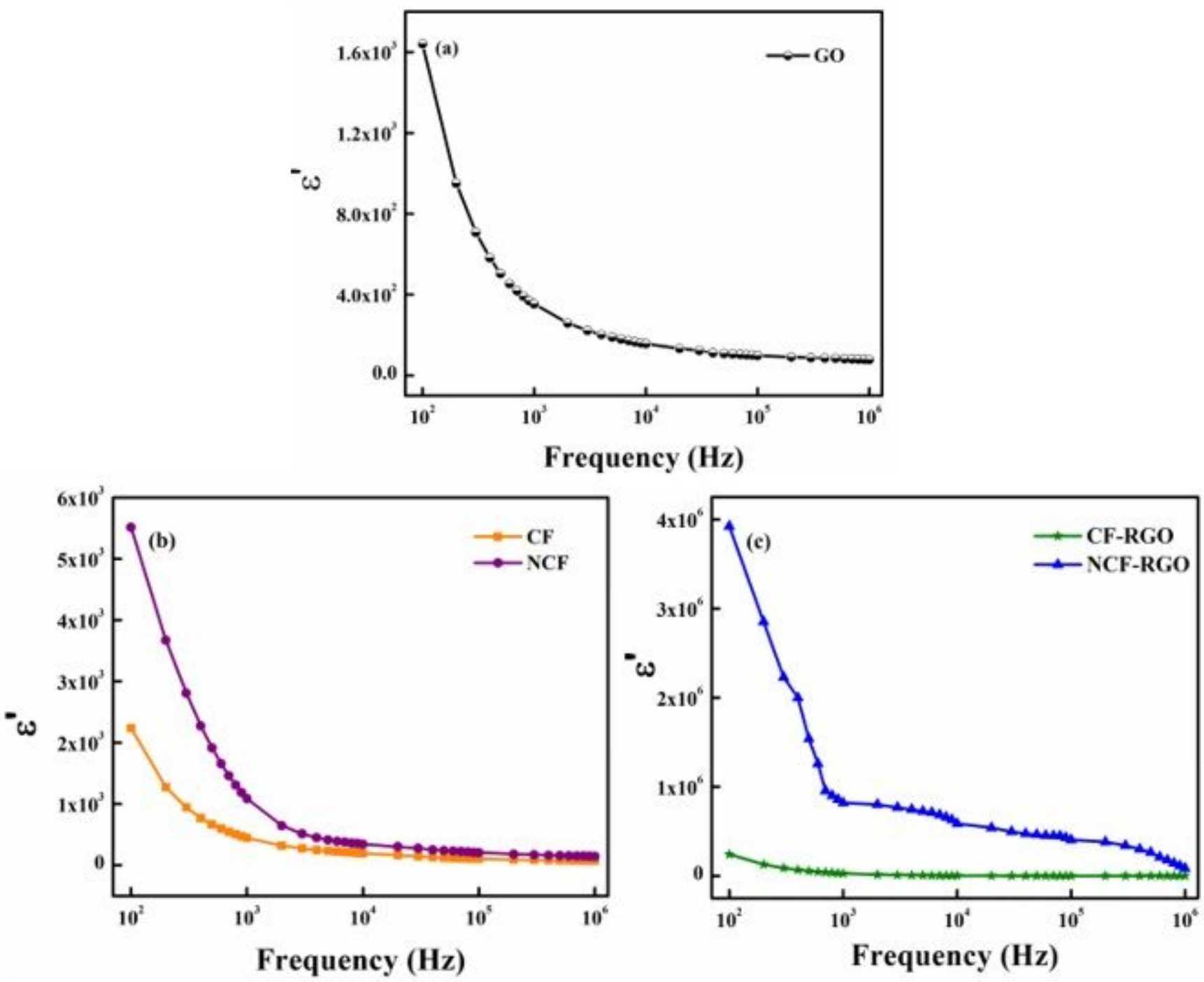

Figure 7

Frequency-dependent $\varepsilon^{\wedge \prime}$ for (a) GO, (b) CF and NCF, (c) CF-RGO and NCF-RGO composites. 

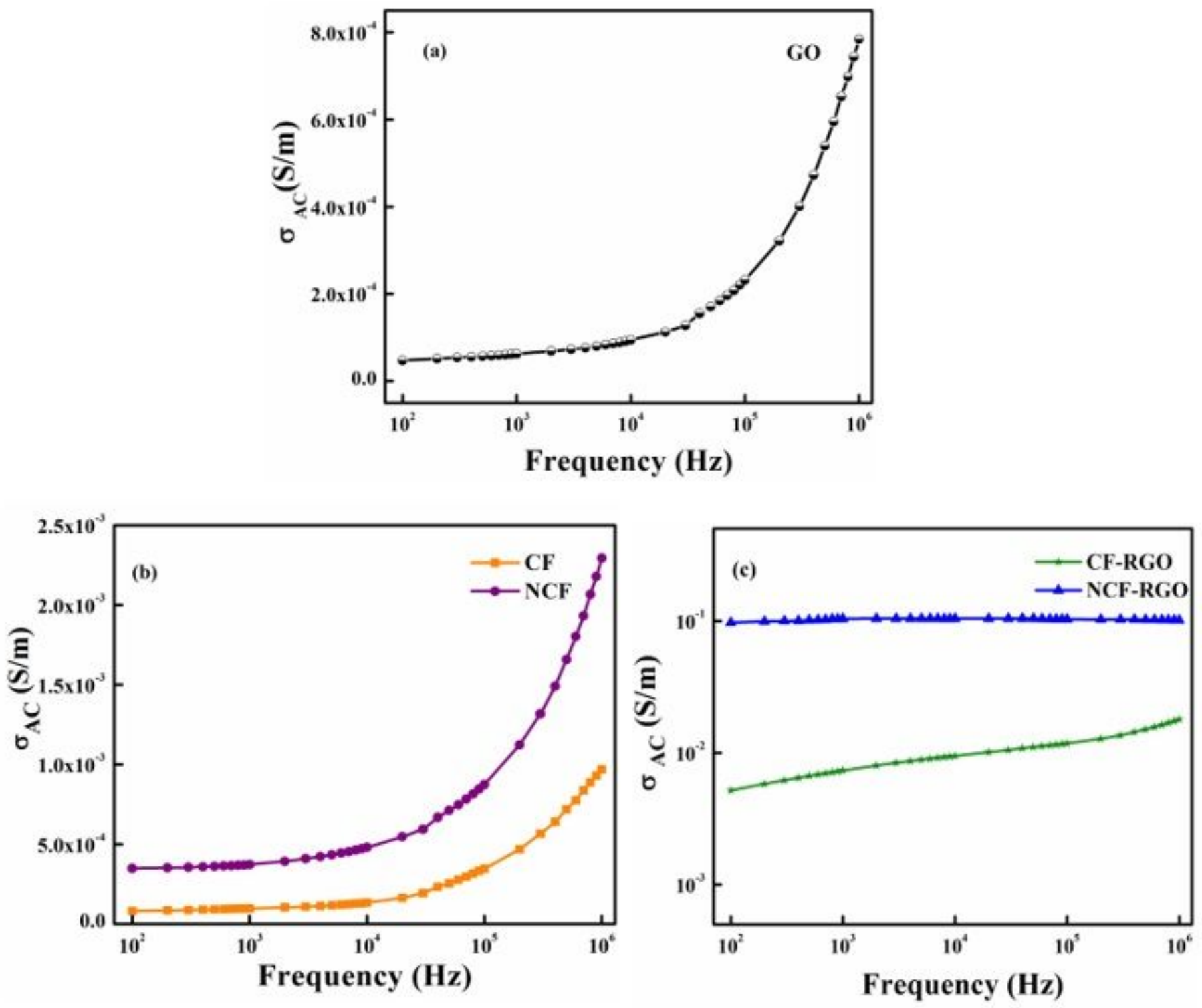

Figure 8

Frequency-dependent $\sigma A C$ for (a) GO, (b) CF and NCF, (c) CF-RGO and NCF-RGO composites. 

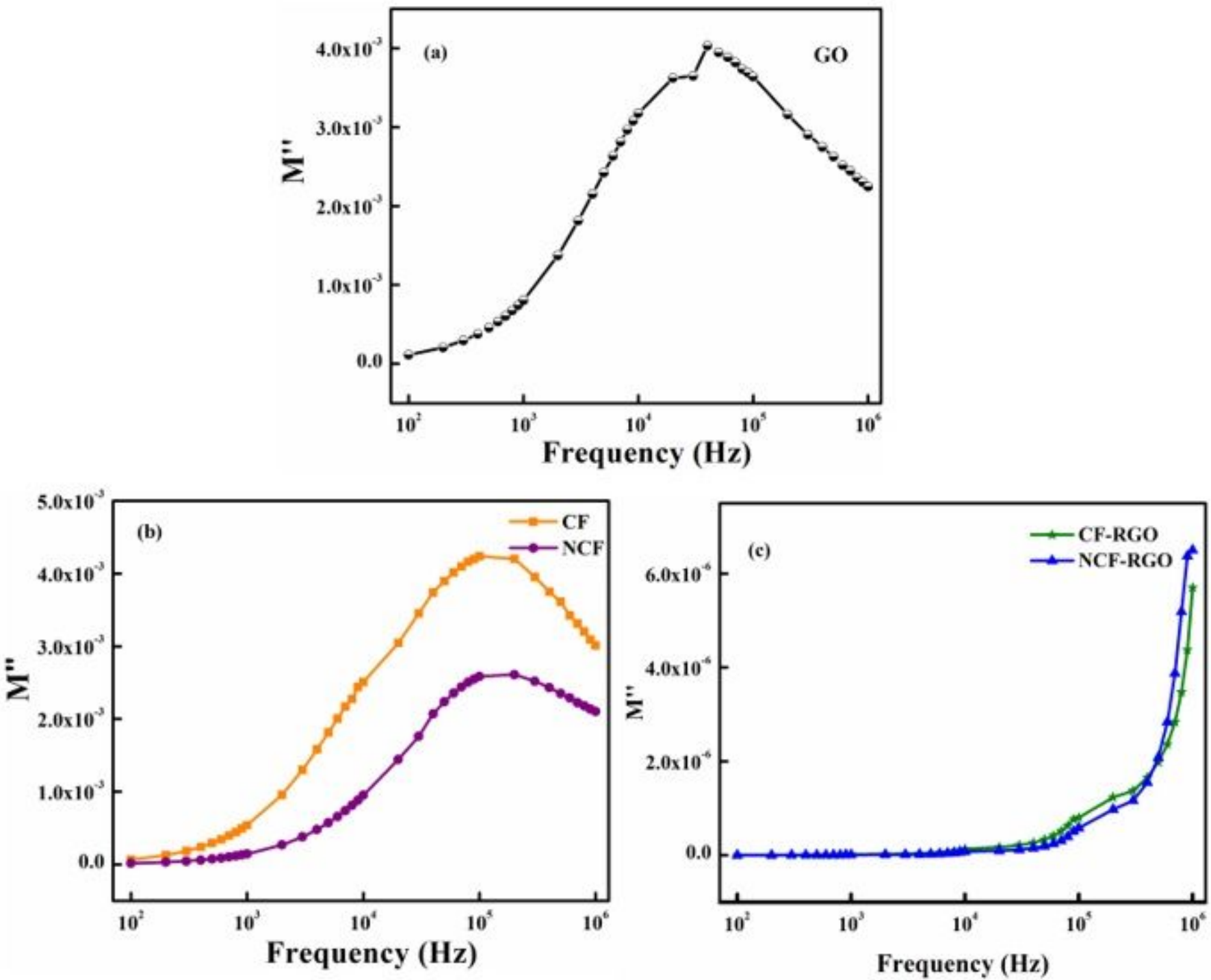

Figure 9

Frequency-dependent $\mathrm{M}^{\wedge}$ " for (a) GO, (b) CF and NCF, (c) CF-RGO and NCF-RGO composites. 

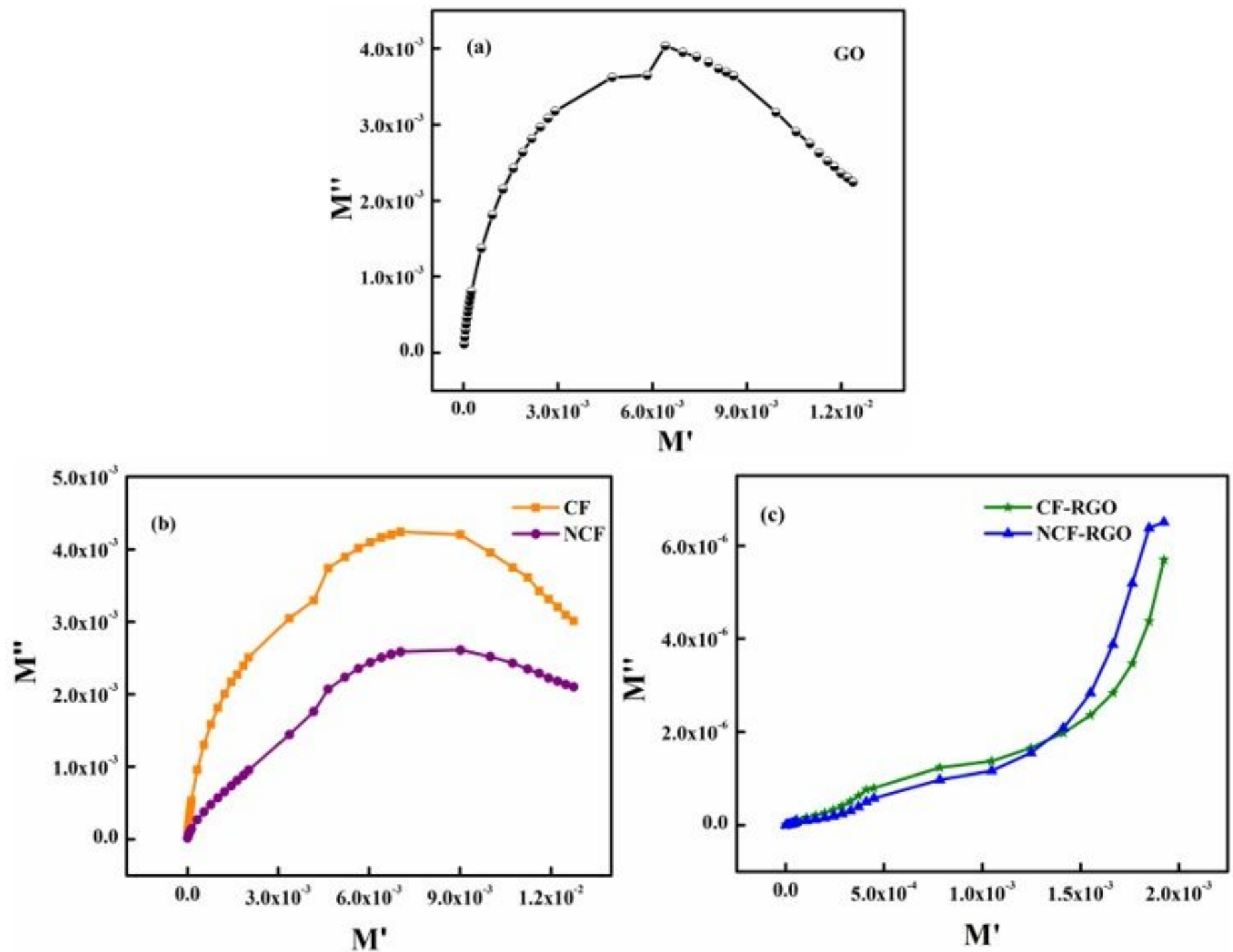

Figure 10

Cole-Cole plot for (a) GO (b) CF and NCF, (c) CF-RGO and NCF-RGO composites. 